\title{
News of BBS
}

\section{Course on Guidelines for Ethical Research with Human Subjects:}

2 weeks course on "Guidelines for Ethical Research with Human Subjects" was organized by BBS in March 2015. Research ethics, Medical mistakes and misconduct, Informed Consent, Conflict of Interest, Fair Subject Selection, Coercion and Undue Inducement, Confidentiality, Vulnerability, Qualitative and Quantitative research; IRB, Preparation for protocol writing, Project proposal for Ethical Clearance; Publication ethics were the topics of lectures.

Prof Dr Akhtarun Naher, Director, NIPSOM; Dr MA Shakoor, Associate Professor, BSMMU; Prof Shamima P Lasker, Secretary General, BBS; Prof M Kabir, Professor of Statistics, Jahangir Nagar University and Prof Ziaul Islam, Professor of Epidemiology, NIPSOM were recourse persons respectively. Ten participants from different medical \& dental colleges attended the course.

\section{Members of BBS in Conferences in Abroad:}

Prof Shamima Parvin Lasker, General Secretary, BBS \& Vice President for South Asia attended the Survey of Research Ethics Committee, Faculty of Medicine, Chiang Mai University, Thailand in March 2015 as a evaluator on behalf of FARCAP.

Dr Arif Hossain, Vice President of BBS attended the International Conference on Building the Collaborative Research Platform and Professional Training Workshop for Cancer Treatment, Hospice/Palliative Care and Bioethics in Southeast Asian Countries in Taiwan in 2014. He presented article "Good Death" which was highly praised by audience. 